\title{
A Numerical Study on a Vertical-Axis Wind Turbine with Inclined Arms
}

\author{
Agostino De Marco, Domenico P. Coiro, Domenico Cucco, and Fabrizio Nicolosi \\ Department of Industrial Engineering (DII), University of Naples Federico II, Piazzale Tecchio 80, 80125 Naples, Italy \\ Correspondence should be addressed to Agostino De Marco; agostino.demarco@unina.it
}

Received 28 August 2013; Revised 5 March 2014; Accepted 2 April 2014; Published 30 June 2014

Academic Editor: W. W. Liou

Copyright (C) 2014 Agostino De Marco et al. This is an open access article distributed under the Creative Commons Attribution License, which permits unrestricted use, distribution, and reproduction in any medium, provided the original work is properly cited.

\begin{abstract}
This work focuses on a particular type of vertical-axis wind turbine, in which a number of inclined arms with airfoil-shaped cross-sections are mounted to connect the principal blades to their hub. While the majority of the known studies on verticalaxis turbines is devoted to the role of principal blades, in most of the cases without taking into account other parts of the wind turbine, the objective of this work is to investigate the effect of uncommon arm geometries, such as the inclined arms. The inclined arms are known to have a potentially beneficial role in the power extraction from the wind current but, due to the complexity of the phenomena, the investigation on aerodynamics of this type of turbine is often impossible through analytical models, such as blade-element momentum theory. It turns out that adequate studies can only be carried out by wind tunnel experiments or CFD simulations. This work presents a methodical CFD study on how inclined arms can be used on a selected wind turbine configuration to harvest additional power from the wind. The turbine configuration, geometry, and some fundamental definitions are introduced first. Then an in-depth CFD analysis is presented and discussed.
\end{abstract}

\section{Introduction}

Due to the rapid consumption of fossil fuel resources and rising general public concerns for sustainable development, wind power generation is actually one of the fastest growing industries for renewable energy production. In addition to large wind farms, studies are focusing on harnessing wind energy in urban and suburban areas.

The two primary types of wind turbine today in use are horizontal axis (horizontal-axis wind turbine (HAWT)) and vertical axis (vertical-axis wind turbine (VAWT)) machines. VAWTs can be further divided into lift-driven (also named Darrieus-type) and drag-driven (named Savoniustype) devices.

HAWTs are highly developed and used in all current large scale wind farms. On the other hand, the majority of research on VAWTs was carried out in the late 1970s and early 1980s, till it became accepted that HAWTs were more efficient at large scales, and interest in VAWTs was dropped out.
Only recently this kind of turbine had undergone renewing interest, because they have indeed a number of advantages over HAWTs: (i) VAWTs are independent on wind direction, so there is no need for a yawing mechanism; (ii) due to the relatively lower rotational speed, VAWTs are typically quieter than HAWTs; (iii) production and maintenance costs of a VAWT can be lower than those for an equivalent HAWT.

On the other hand, a VAWT has an inherently nonstationary aerodynamic behavior due to the continuous variation of incidence angle and relative velocity experienced by the blades during a complete revolution. This leads, especially at low angular speed (low tip speed ratios), to the dynamic stall phenomenon that has significant effects on unsteady loads acting on the blades and consequently on turbine performance. Moreover, every half rotation each blade actually works in its own wake as well as in other blades wakes, further enhancing the nonstationary behavior of the motion.

Due to the complexity of the phenomena, the investigation of VAWT aerodynamics is often impossible through 


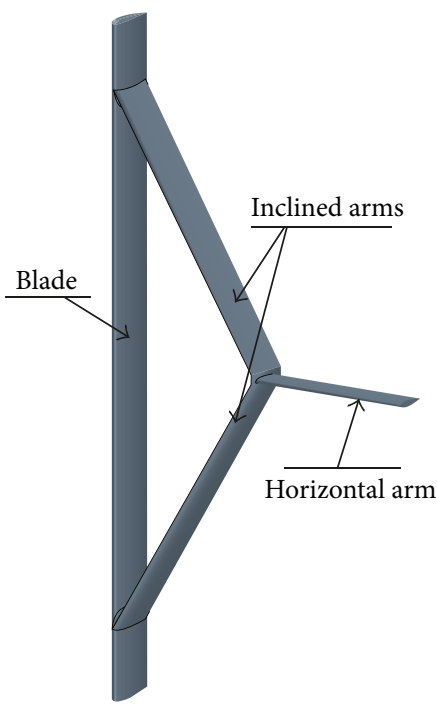

(a)

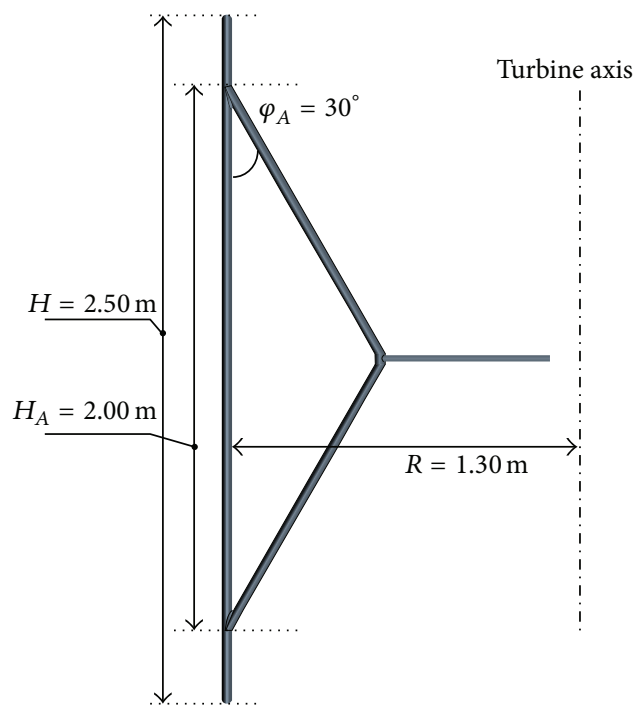

(b)

FIgURE 1: Parts and dimensions of one element of the proposed vertical-axis wind turbine with inclined arms.

analytical models, such as blade-element momentum theory [1]. It turns out that adequate studies can only be carried out by wind tunnel experiments or CFD simulations.

Recently, several authors [2-4] performed both 2D and 3D numerical simulations of Darrieus micro-VAWTs. Simão Ferreira et al. [5] compared the results of two types of RANS solutions (with Spalart-Allmaras [6] and K-Omega [7] turbulence models) and of large Eddy simulation (LES) and detached Eddy simulation (DES) with experimental vorticity visualization for a single-bladed VAWT. Castelli et al. [8-10] presented a fairly complete numerical study of the effect on turbine performance of different blade parameters such as airfoil asymmetry, blade inclination, and number. The same authors [11] proposed also an interesting turbine performance prediction model. Amet et al. [12] performed accurate 2D numerical studies on the mechanism of vorticity generation in VAWTs. Hwang et al. [13] presented numerical simulations of blade pitch control systems.

While the majority of the known studies on VAWT focus on the blade itself, in most of the cases without taking into account other parts of a wind turbine, the present work objective is to investigate the effect of uncommon arm geometries of VAWT, such as the "inclined arms" (see Figure 1). Some phenomena occurring when this configuration rotates in the wind are still not completely understood nowadays. This offers the chance to investigate on how the inclined-arms VAWT can be used to harvest additional power from the wind. The Aircraft Design and AeroflightDynamics Group of the University Federico II Department of Industrial Engineering (Aerospace Division) have developed several prototypes of this kind of HAWT and VAWT [14-16]. Initial estimations obtained with vortex methods have shown good results, encouraging further investigation of this kind of turbine. The present work reports a CFD analysis of selected turbine geometry with this concept.
In the next sections the turbine configuration, geometry, and some fundamental definitions are introduced first. Then an in-depth CFD study realized for the present work will be presented. Finally, the principal results obtained will be discussed.

\section{Inclined-Arms Turbine Configuration}

As depicted in Figure 1, the geometry of the inclined-arms concept implies the presence of two inclined arms with airfoil-shaped cross-sections. The inclined arms are joined to a horizontal arm that connects the blades to the hub. There are two main advantages stemming from this particular configuration:

(i) the inclined arms act as additional blades; the net torque provided by the arms per revolution at least neutralizes their own parasite torque (due to their drag); and, as will be shown, additional power can be effectively extracted;

(ii) a better distribution of mechanical loads on blade attachments with respect to horizontal-arms turbines.

The complete turbine configuration consists of three rotating "elements" mounted on the hub. In the study presented here, each element is formed by

(i) a NACA 0018 straight, untwisted vertical blade, with $20 \mathrm{~mm}$ of chord and $5^{\circ}$ of pitch (outwards with respect to the circular path of rotation, see Figure 2(b));

(ii) 2 inclined arms whose cross-section has the same profile of the vertical blade; their external tip is attached at some point along the vertical blade; and each inclined arm forms a $30^{\circ}$ angle with the blade;

(iii) an inner horizontal arm, with NACA 0018 profile and $12 \mathrm{~mm}$ of chord, connecting inclined arms to the hub. 


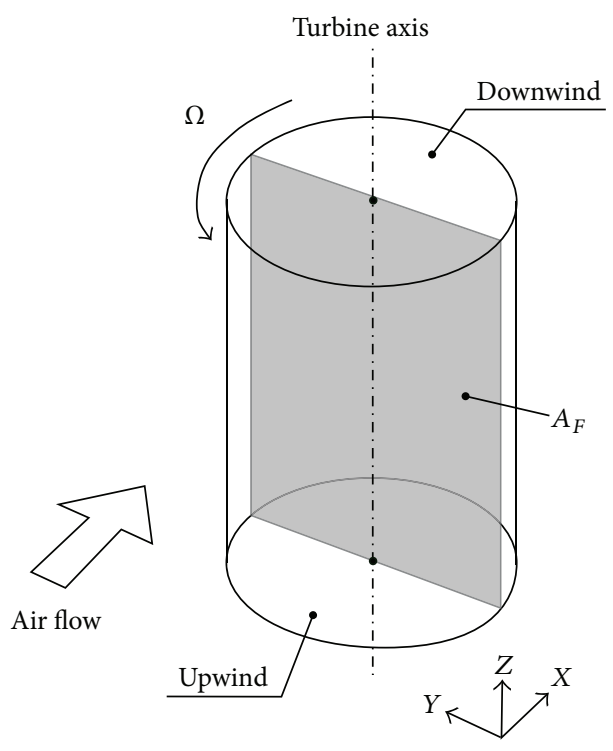

(a)

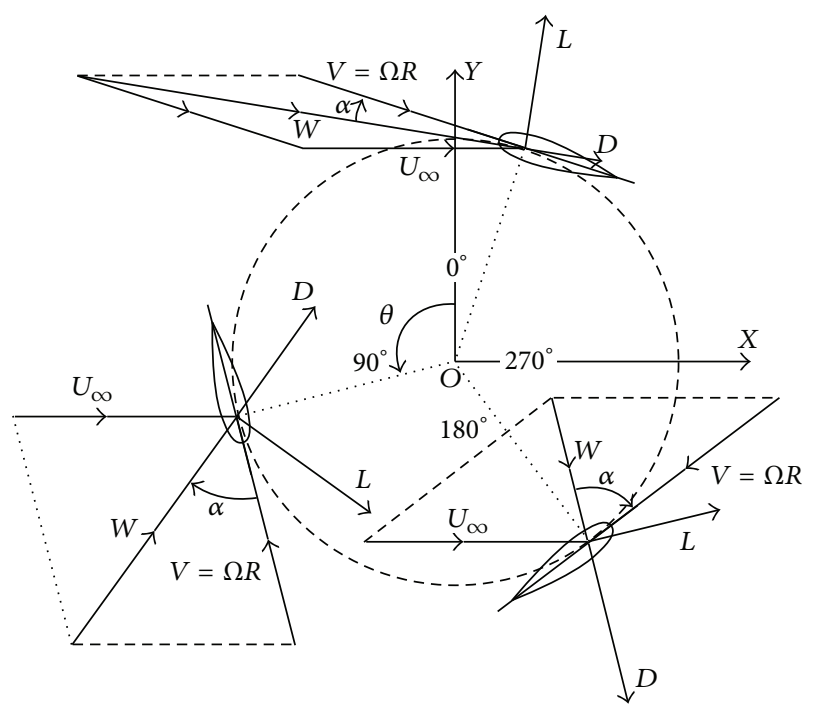

(b)

Figure 2: (a) Conceptual visualization of a VAWT. (b) Forces and velocities on a Darrieus turbine [5].

\section{Turbine Parameters}

For the discussion of results presented later in this work, some fundamental definitions have to be established. Turbine solidity is defined as follows:

$$
\sigma=\frac{n c}{R}
$$

where $n$ is the number of blades and $c$ is the blade chord. The tip speed ratio $\lambda$ (or TSR) is given by

$$
\lambda=\frac{\Omega R}{U_{\infty}},
$$

where $\Omega$ is the turbine angular velocity and $U_{\infty}$ is the wind velocity.

From geometric considerations, theoretical relative velocity modulus $W$ and incidence angle $\alpha$ are given by

$$
\begin{gathered}
W=U_{\infty} \sqrt{1+2 \lambda \cos \theta+\lambda^{2}}, \\
\alpha=-\tan ^{-1}\left(\frac{\sin \theta}{\cos \theta+\lambda}\right),
\end{gathered}
$$

where the angle $\theta$ is the azimuthal position of the element along the revolution. The azimuthal position is zero when the element is facing the asymptotic wind direction and grows as the blade rotates counterclockwise (see Figures 2, 3, and 4).

The sign convention of the blade incidence angle $\alpha$ is the same of the blade pitch angle $\beta$, which is considered positive when the blade leading edge is rotated outwards with respect to the circular path of rotation. Then, when the azimuthal position is zero, the incidence angle is positive if the blade has a positive pitch.

The results discussed later in this work will be shown in terms of the turbine performance coefficient (or power coefficient) $C_{P}$, which is calculated from the torque $T$ as follows:

$$
C_{P}=\frac{T \Omega}{(1 / 2) \rho U_{\infty}^{3} A_{F}},
$$

where $A_{F}$ is the rotor frontal area ( $2 R$ times the blade height h).

\section{A CFD Study on an Inclined-Arms VAWT}

The study presented here goes straight to the numerical calculation of the three-dimensional fluid dynamic field around the rotating inclined-arms wind turbine. The analysis cannot evolve from preceding two-dimensional simulations due to the intrinsic three-dimensional characteristics of this particular rotor.

The computational fluid dynamics code used in our numerical studies is Star-CCM+ by CD-Adapco. It solves the Reynolds-averaged Navier-Stokes (RANS) equations over a properly designed three-dimensional computational mesh. The turbulence model chosen is the SST K-Omega [7].

The simulation domain is divided into two regions:

(i) a stationary block-shaped outer zone, determining the extent of the overall calculation domain;

(ii) an inner rotating block that contains a cylindrical rotating subdomain centered on turbine axis; in this moving domain the mesh is finer than the outer mesh and conforms to the turbine shape.

The six sides of the outer region have different boundary conditions (BCs). A velocity Inlet BC is placed upwind at distance $10 R$ from the turbine axis. A pressure outlet was placed downwind at distance $15 R$, allowing a full development of the wake. Lateral sides of the computational domain are placed 


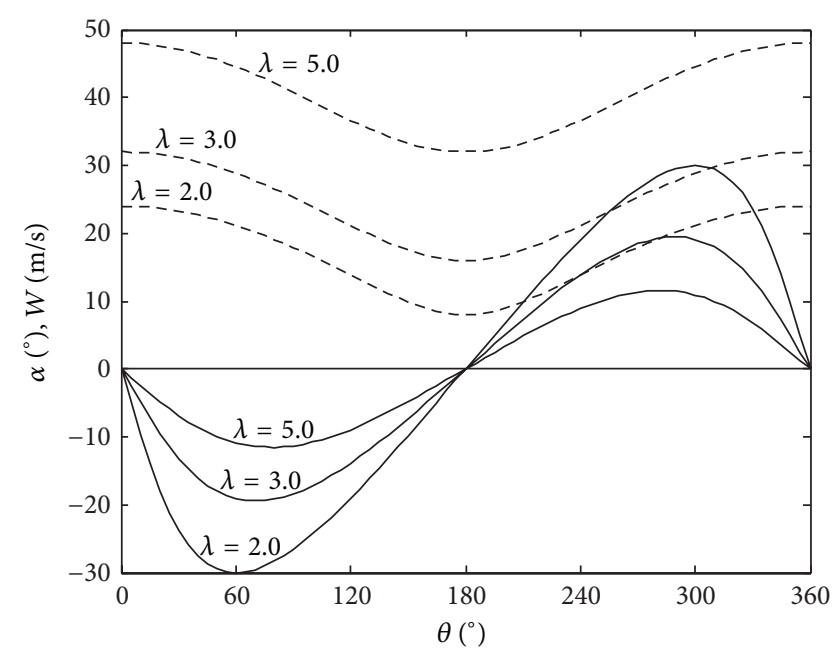

FIGURE 3: Incidence angle $\alpha$ (solid lines) and relative velocity magnitude $W$ (dashed lines) during a turbine revolution for different tip speed ratios, with no blade pitch.

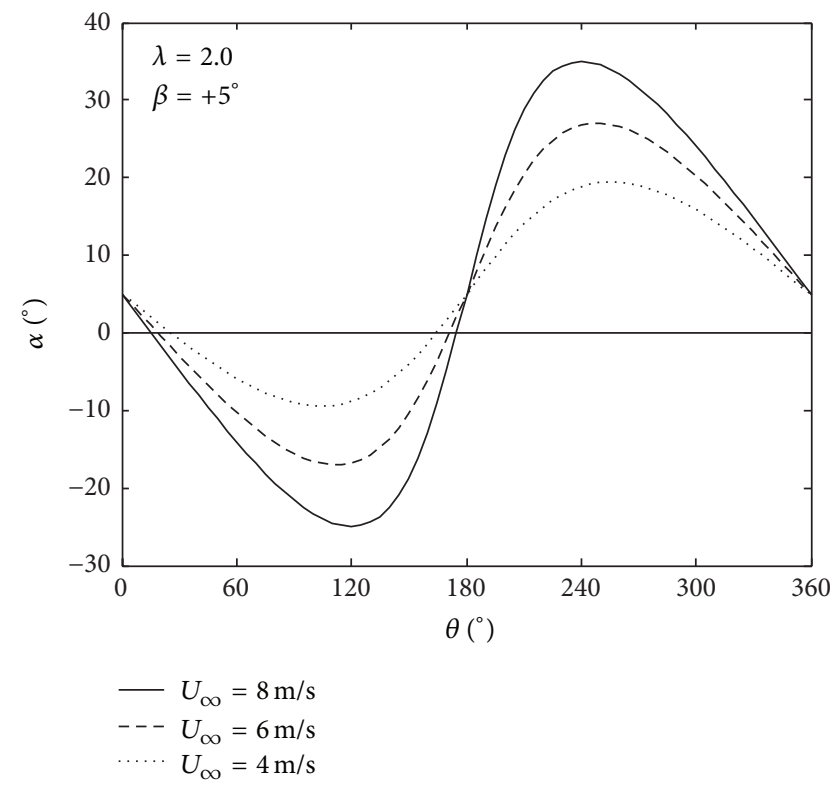

FIGURE 4: Incidence angle during a turbine revolution for different wind velocities, with fixed tip speed ratio and $5^{\circ}$ positive blade pitch (curves shift upwards).

$10 R$ away (left and right) from the axis. Top and bottom sides are placed $5 R$ from the middle of the hub. Left, right, top, and bottom sides are all assigned with Slip Wall BCs. See Figure 5 for the computational domain geometry.

The computational grid is unstructured ("trimmed" according to the Star-CCM+ definitions [17]) and is formed almost entirely of hexagonal cells, except near the solid walls, inside the inner cylindrical domain, where finely meshed prismatic layers are used to model the boundary layer regions (see Figure 6). The thickness of the near-wall prism layer was chosen to achieve wall $\mathrm{y}^{+}$values around 1 , corresponding to a size of $10 \mu \mathrm{m}$, while the total extension of the prism layers is set adequately larger than the expected thickness of the local turbulent boundary layer. Grid had been finely enhanced in critical areas such as leading and trailing edges, corners, and turbine wake.

A grid sensitivity study was performed to achieve mesh independence. This resulted in $3 \mathrm{D}$ models whose number of cells ranges between 4 and 18 million, according to the number of turbine elements simulated.

The velocity inlet boundary allows the magnitude of inlet flow and turbulent quantities to be specified. Wind velocity is set to $8.0 \mathrm{~m} / \mathrm{s}$. The turbulent intensity applied is $20 \%$ according to IEC international standard for wind turbines [18]. Turbulence velocity and length scale are, respectively, equal to wind velocity and typical cell dimension in the direction of the flow.

Initial simulations used a temporal discretization chosen to satisfy Courant-Friedrichs-Lewy condition [17], which limits the time-step in order to obtain a unitary value of the CFL number. The asymptotic velocity and the dimensions of the smallest grid cell outside of the prism layers (inside the boundary layer velocities are smaller and decreasing concurrently with cell dimensions) were used to calculate the time-step, which was somewhere about $0.1 \mathrm{~ms}$. Then, several simulations were carried out subsequently increasing the time-step. Since these simulations did not show any significant variation, the final time-step was set equal to the lapse of time the rotor takes to make a $1^{\circ}$ rotation (around $1 \mathrm{~ms})$.

\section{Code Validation}

The results discussed later were validated by comparisons with experimental data. A turbine rotor whose geometry is very similar to the one presented was mounted onto a truck and tested in open-field trials (see Figure 7). The rotor tested experimentally has a slightly higher radius with respect to the one used in numerical simulations (1.43 against 1.30 meters). The performance coefficients obtained both numerically and experimentally in selected working conditions are plotted in Figure 8.

The numerical results show a good agreement with the experimental data. Both the curve slope and the TSR corresponding to the maximum performance coefficient are captured. Some minor discrepancies between numerical and experimental data are probably due to the discrepancies between the physical models employed in the simulations and the real conditions experienced by the turbine.

However, this work's objective is to focus less on the exact numerical values and more on performance trends as the geometric parameters change, and then this level of accuracy was considered appropriate.

\section{Results and Discussion}

Simulations were performed on the university supercomputing infrastructure S.Co.P.E. [19], which hosts a cluster of about 300 eight-core servers. A typical simulation is 


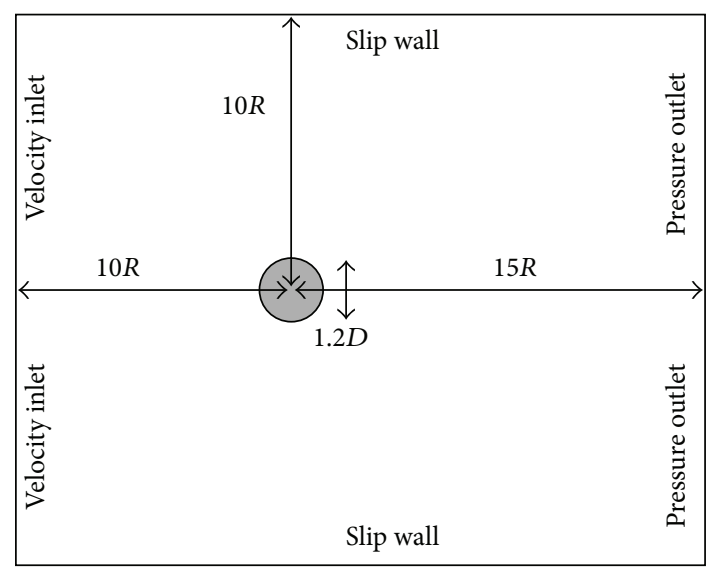

(a)

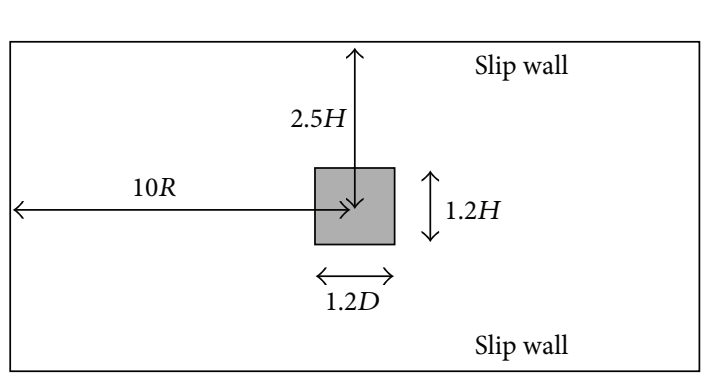

(b)

Figure 5: Upper view (a) and frontal view (b) of computational domain. The rotating region (in grey) inside the stationary one can be seen, along with their dimensions with respect to turbine radius $R$, diameter $D$, and height $H$. Different boundary conditions are also shown.
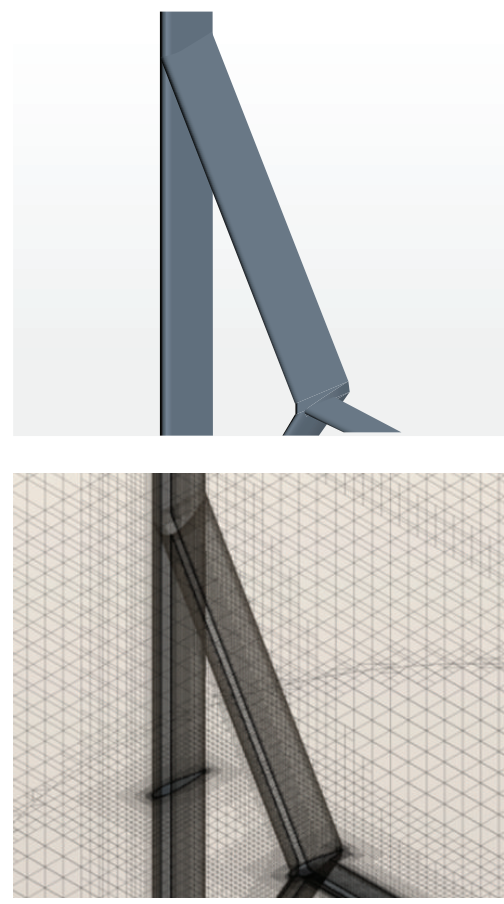
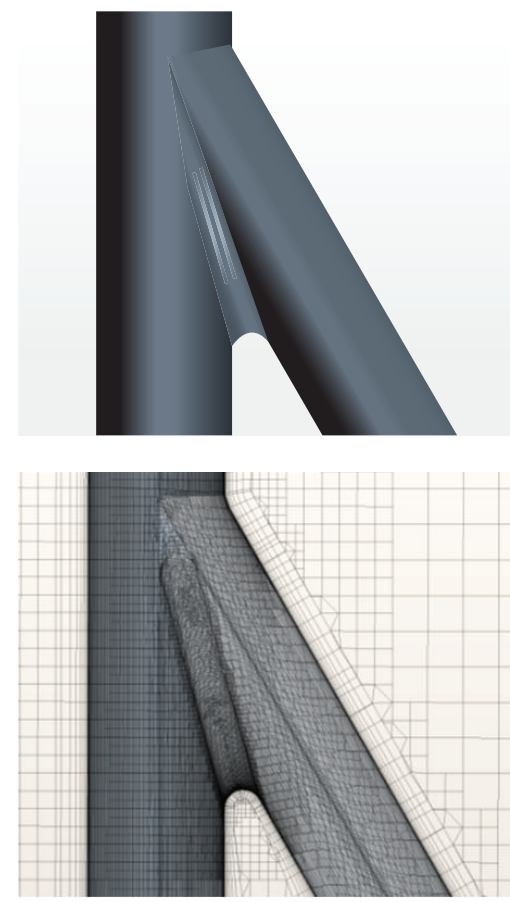
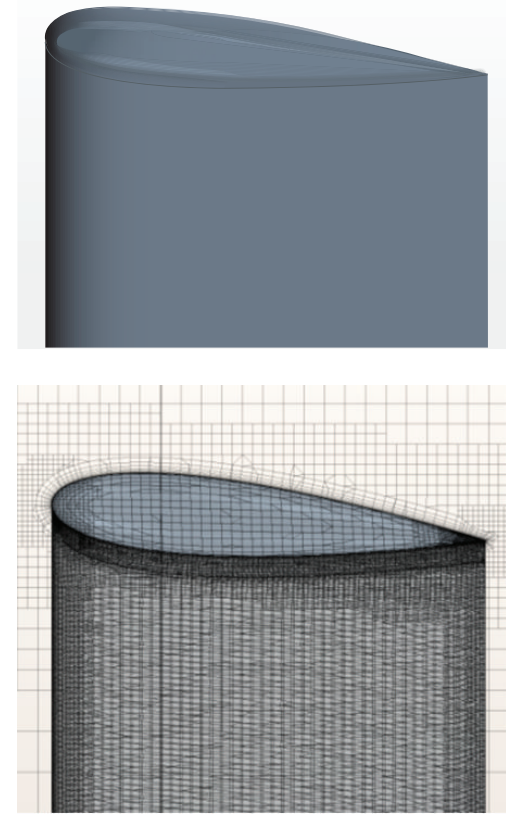

FIGURE 6: Turbine parts close up both of the CAD model and the final mesh; the latter visualized also with the mesh of the open space surrounding the turbine.

performed on 64 cores and takes about 24 hours of computing time to fulfill one turbine revolution.

When doing this kind of simulations an initial transient behavior of the numerical solution has to be expected. Typically the transient covers at least the first two revolutions. This behavior was noticed in the simulations presented here. Significant differences in the flow field are present between the first and the second revolution; then these differences become less evident and negligible for the successive revolutions. The results presented below correspond to the 5 th revolution, when the wake is completely developed downstream.

\section{Single Element Efficiency}

The first part of this study focuses on results obtained for the turbine "single element." Here a single element is intended as the single blade with the two arms (see Figure 9, first row). The aim of this initial study is to assess the single element efficiency when it is not influenced by the presence of other elements. In particular, three different simulations were carried out:

(i) a simulation concerning one vertical blade alone (IB$1)$; 

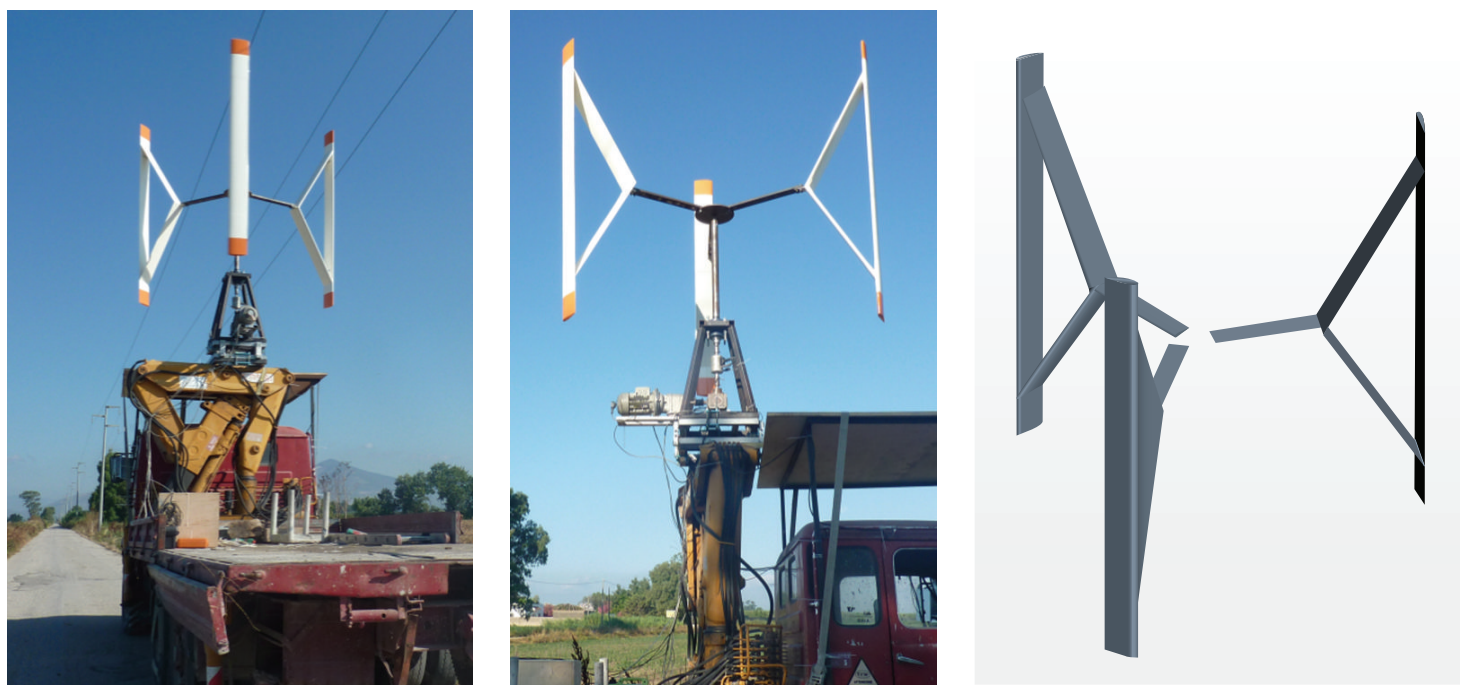

FIGURE 7: Real turbine during experiments performed in open field, along with the turbine geometric model used in the simulations.

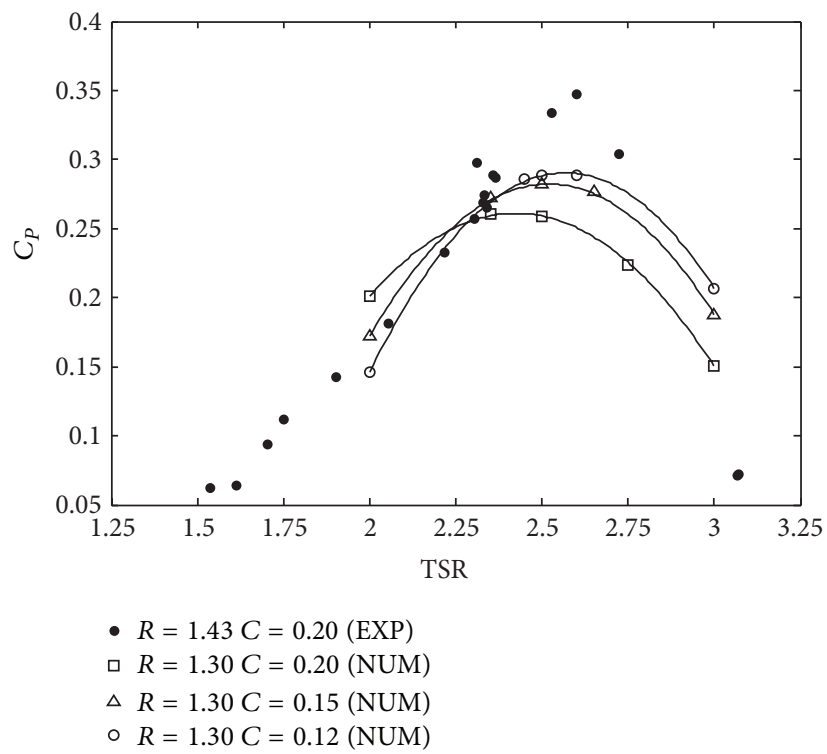

Figure 8: Turbine performance coefficients as a result of experimental (EXP) and numerical (NUM) data. Numerical analyses were performed on several geometries (radius $R$ and inclined arm chord $C$ in meters).

(ii) a simulation concerning one set of inclined arms alone (IA-1);

(iii) a simulation of the full element (FE-1, blade plus the two inclined arms).

It has to be observed that the horizontal arms connecting the inclined arms to the hub have been modeled as well to account for their wake effects in the simulations. However, their parasite torque was not considered in the analyses presented in Figure 10(a) and Table 1(a).

Figure 10 shows the instantaneous torque $T$ as a function of the azimuthal position $\theta$ provided by the isolated blade (IB-1), by the isolated couple of inclined arms (IA-1), and by the full configuration (FE-1). The theoretical curve (THEOR) given by the algebraic sum of IB-1 and IA-1 is also shown.
This latter curve is named "element theoretical torque," in opposition to the "element actual torque" (FE-1). It turns out that, as expected, the actual torque average value $T_{\text {avg,FE }}$ is lower than the theoretical torque average value $T_{\text {avg,THEOR. In }}$ particular, $T_{\text {avg,FE }}$ is about $14 \%$ less than $T_{\text {avg,THEOR. Despite }}$ this fact, the actual average torque is still quite larger than the one provided by the vertical blade alone, $T_{\text {avg,IB }}$.

Similarly, the average values per revolution of the performance coefficient $C_{P}$ are compared in Table 1(a) for the various cases IB-1, IA-1, THEOR, and FE-1. Average values in the upwind and downwind zones are also shown in the same table. The "upwind zone" contribution is the average of instantaneous $C_{P}$ over the range $0^{\circ} \leq \theta<180^{\circ}$. The "downwind zone" contribution is the average over the range $180^{\circ} \leq \theta<360^{\circ}$. 


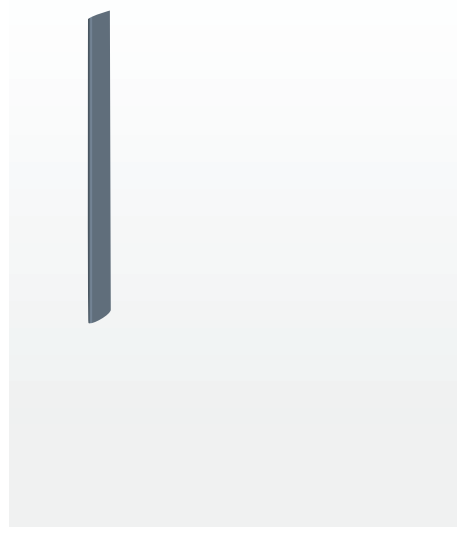

1 isolated blade (IB-1)

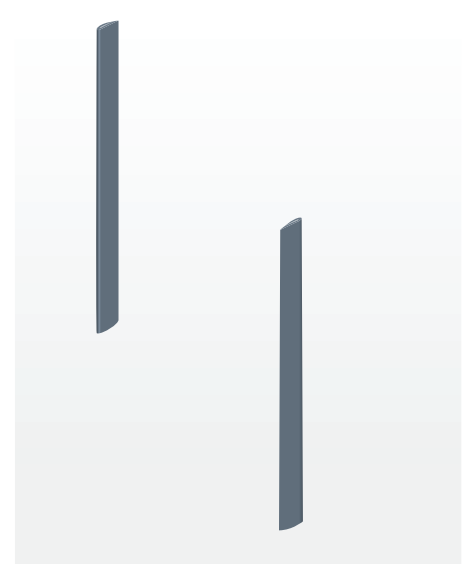

2 isolated blades (IB-2)

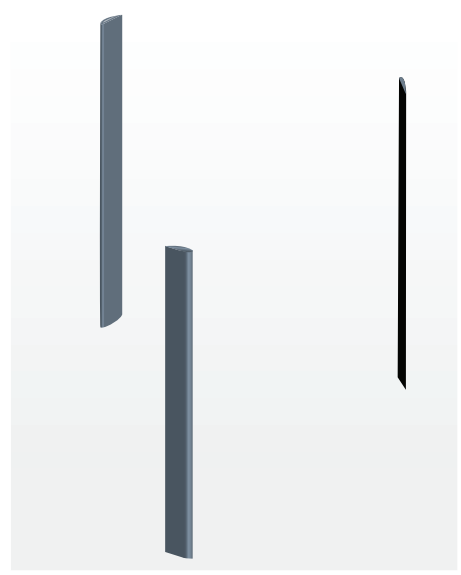

3 isolated blades (IB-3)

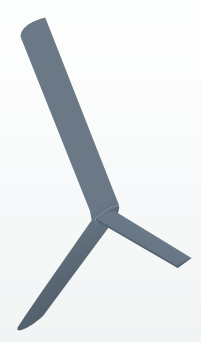

1 isolated set of arms (IA-1)

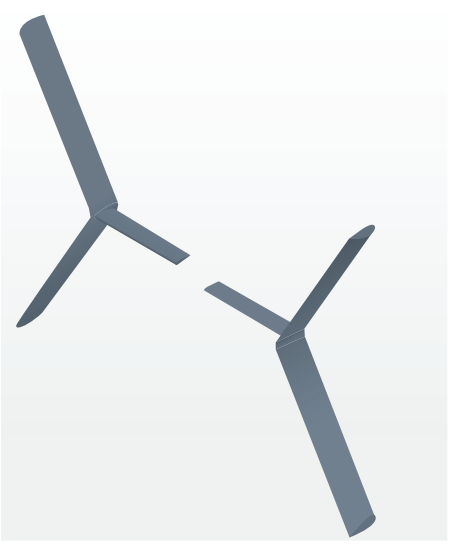

2 isolated sets of arms (IA-2)

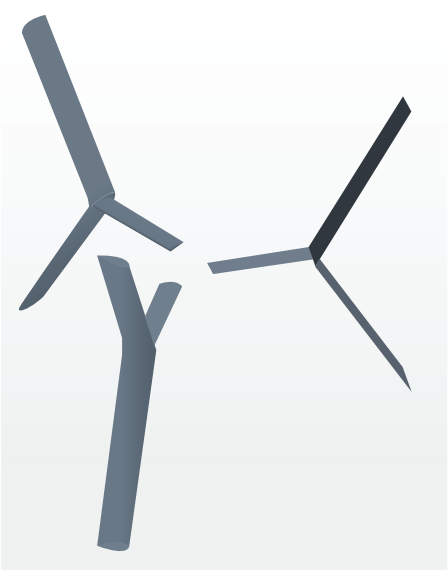

3 isolated sets of arms (IA-3)

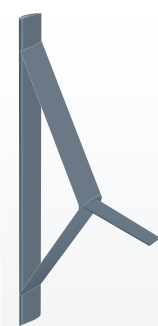

1 full element (FE-1)

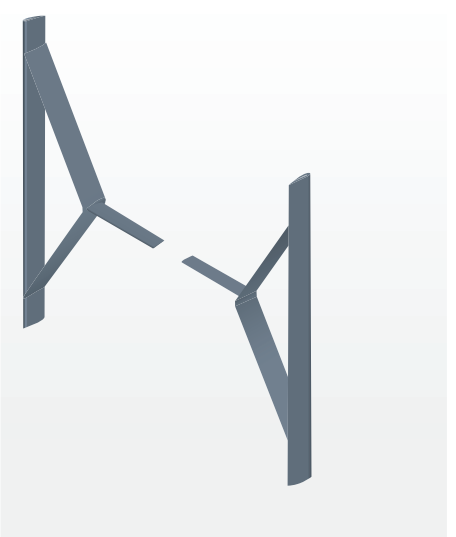

2 full elements (FE-2)

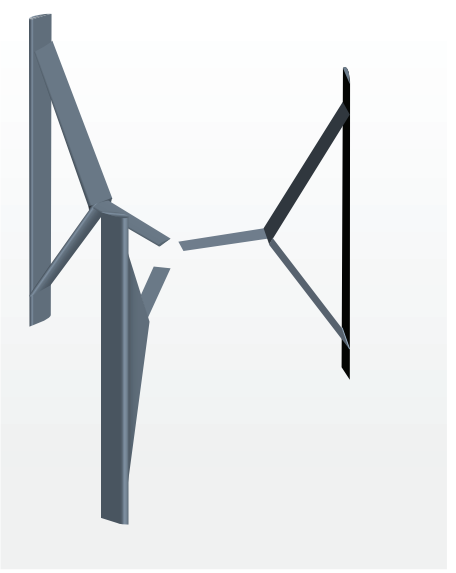

3 full elements (FE-3)

FigURE 9: Isolated blades, isolated sets of inclined arms, and full elements analyzed in the simulations.

A fact that stems from Figure 10(a) and Table 1(a) is that the torque reduction from the theoretical case (Theor) to the actual case (FE-1) takes place mainly in the upwind part of the revolution, where the average torque is reduced by about $17 \%$. In the downwind zone, instead, the actual torque is less negative (less parasitic) than the theoretical one. In this respect, it has to be observed that the entire turbine element (case FE-1) generates a larger wake with respect to the wakes generated by the isolated blade (case IB-1) and the isolated set of arms (case IA-1). Figure 10(b) shows a vorticity map comparison for the IB-1 and FE-1 configurations. The vertical plane where the vorticity magnitude is mapped is placed 


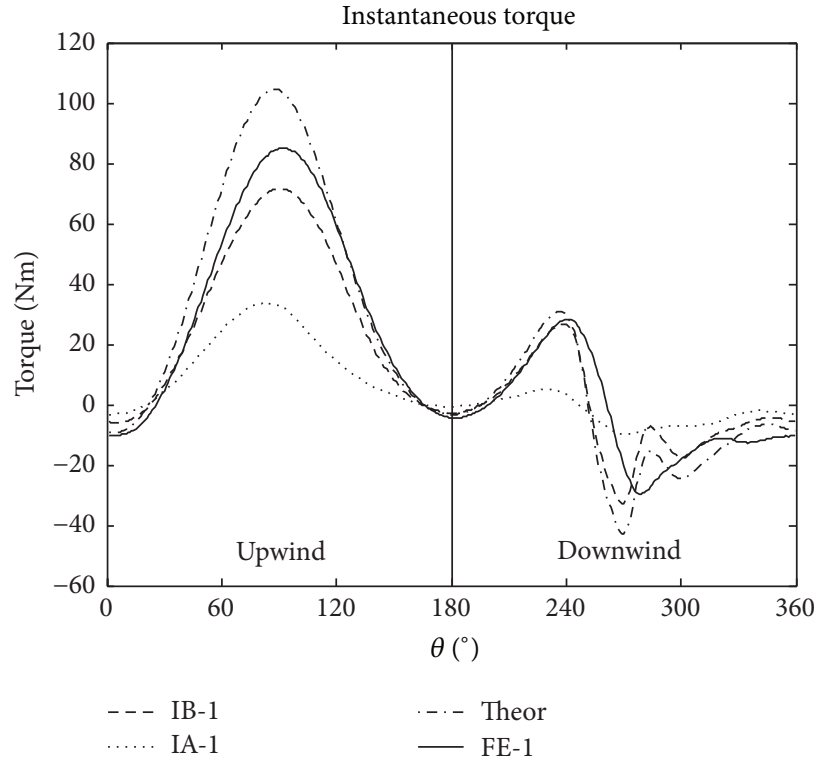

(a)
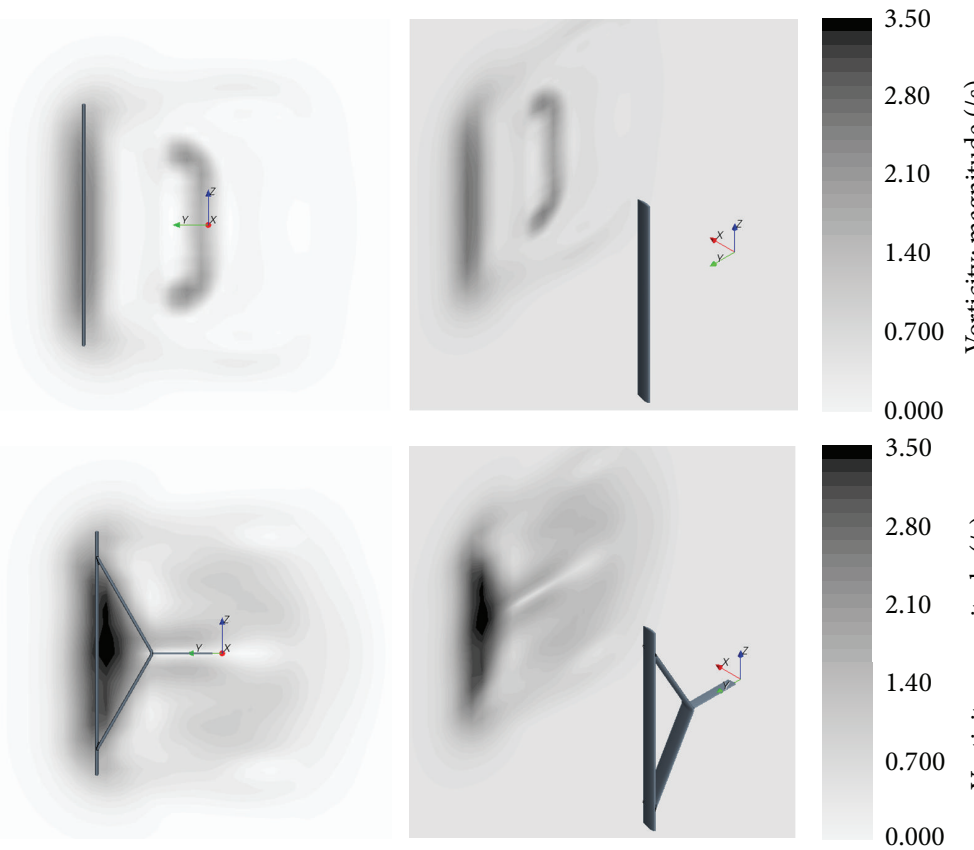

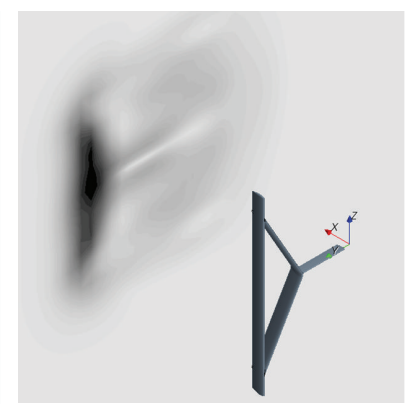

(b)

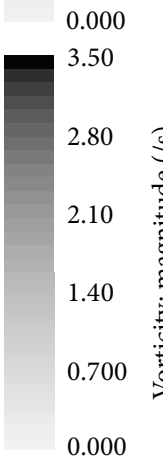

0.000

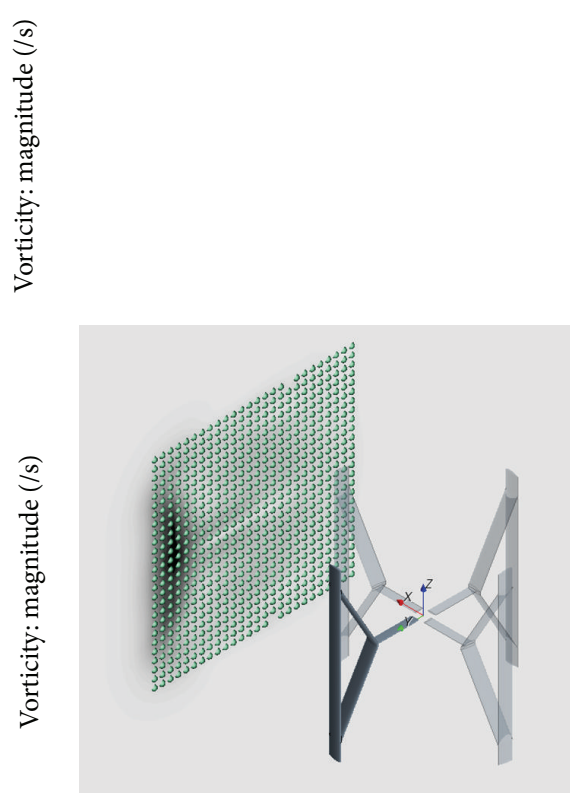

(c)

FIGURE 10: (a) Instantaneous torque generated during one revolution by one isolated blade (IB-1) and one isolated set of arms (IA-1), along with theoretical (arithmetical sum of blade and arms torques, IB-1 plus IA-1) and actual (FE-1) entire element results. (b) Comparison of vorticity magnitude maps in a plane behind IB-1 (top) and FE-1 (bottom) configurations. (c) Vorticity in a plane behind the FE-1 configuration (free stream coming from lower right). The rotating element is shown in four subsequent positions during one revolution and the dots represent the grid used for the vorticity maps (see Figure 10(b)) and the quantitative evaluation of wake strength (see Table 1(b)).

behind the rotor orthogonally to the free stream velocity (see Figure 10(c)). The high values of the vorticity magnitude in the area immediately behind the "triangle" made by the vertical blade and the two inclined arms (see Figure 10(b), bottom left) give an idea of the stronger wake of the case FE-1 over the case IB-1. Average values of the vorticity magnitude are reported in Table $1(\mathrm{~b})$.
During an entire revolution the rotating element encounters its own wake and one effect of this wake is to reduce the local asymptotic airspeed seen by the blade profiles, decreasing the local incidence angles (see Figure 4). The positive pitched blade experiences larger incidence angles in the downwind zone and a greater reduction of these large angles has a positive effect on the blade instantaneous torque. 


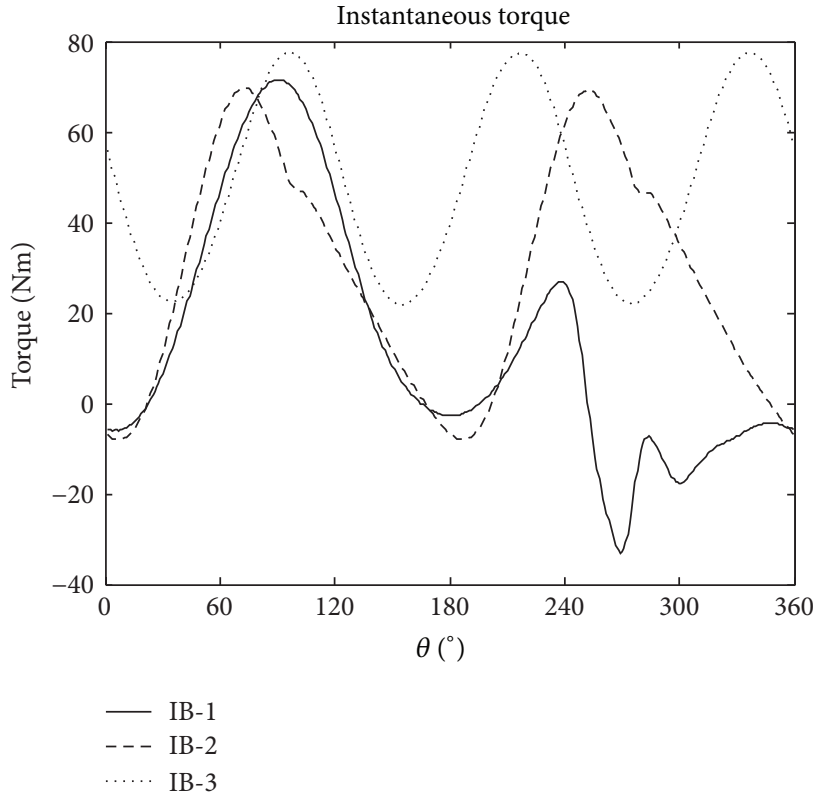

(a)

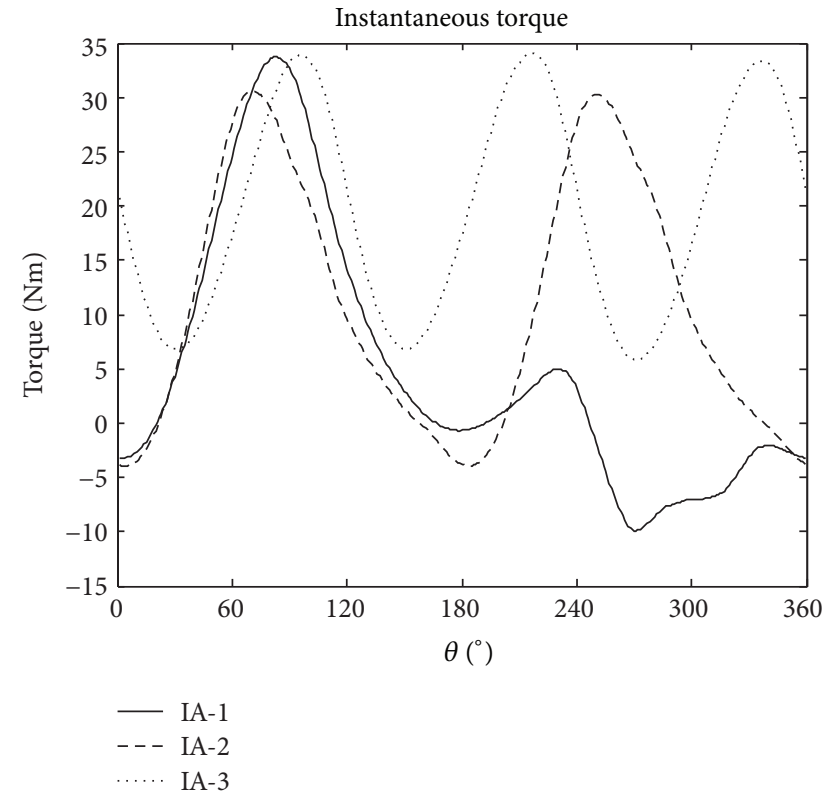

(b)

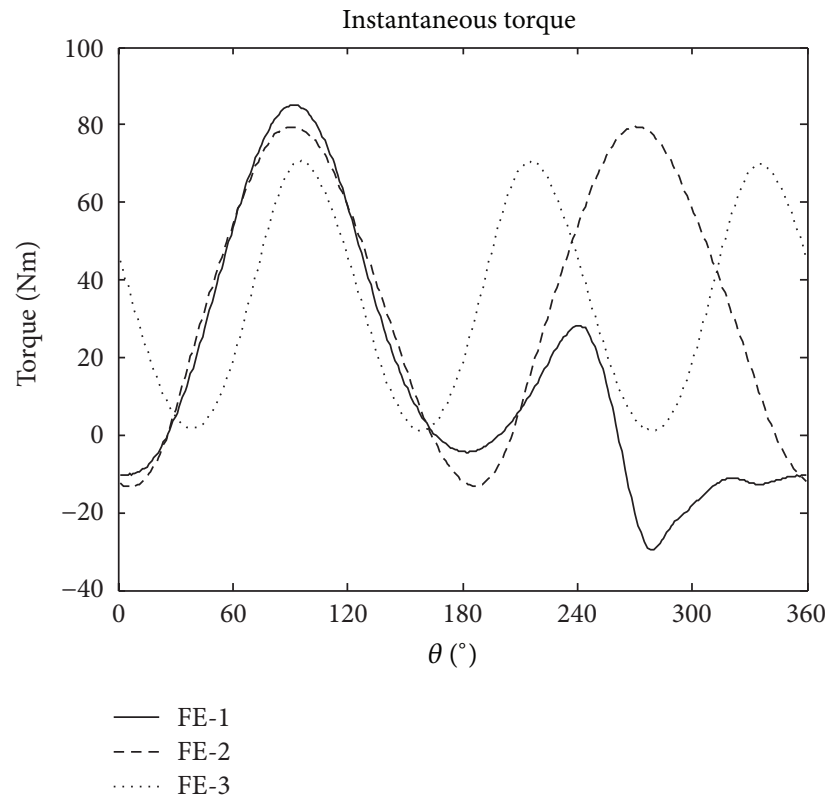

(c)

FIGURE 11: (a) Instantaneous total torque when a different number of isolated blades are present in the simulation. (b) Instantaneous total torque when a different number of isolated set of arms are present in the simulation. (c) Instantaneous total torque when a different number of full elements are present in the simulation.

On the other hand, in the upwind zone, the local incidence angles are already small and the reduction introduced by a stronger wake (case FE-1) has an evident negative effect.

\section{Multiple Elements Efficiency}

In successive analyses the turbine element (FE-1, one blade plus one set of arms) is first doubled, obtaining a two-element rotor. Then the real turbine composed of three elements is finally considered. Also in these cases the investigation involves first the isolated couple, or triplet, of blades (IB-2 or IB-3), and the isolated couple, or triplet, of arm sets (IA-2 or IA-3). This is aimed at identifying the beneficial sources of the full rotor efficiency. These simulations correspond to the cases depicted in the second and third rows of Figure 9.

As shown in Figure 11, increasing the number of single parts (blades only, or arms only) or elements corresponds to increasing the number of peaks in the instantaneous torque, 

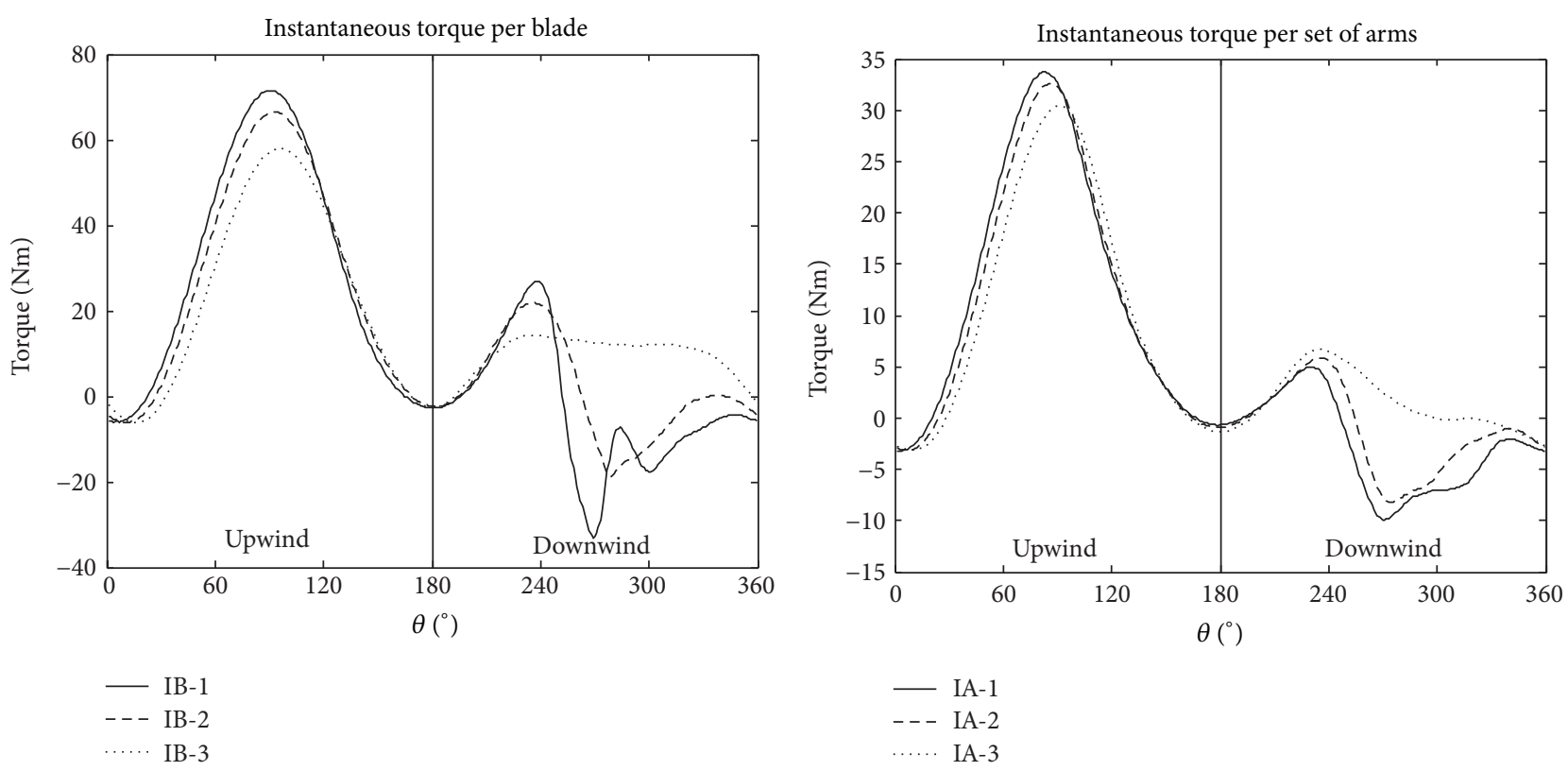

(a)

(b)

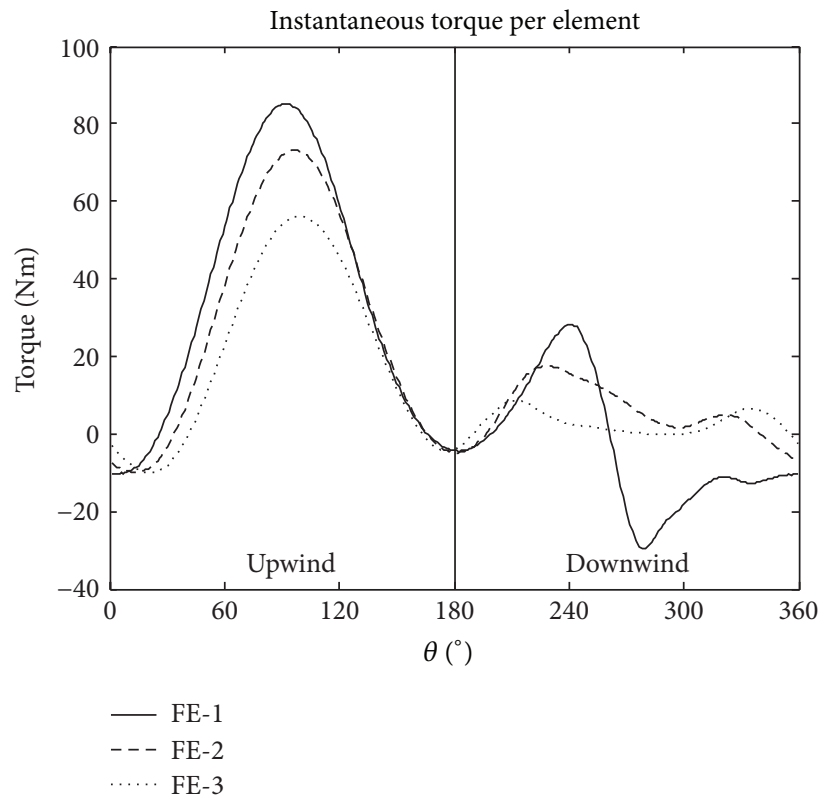

(c)

FiguRE 12: (a) Instantaneous torque per blade (generated by a single isolated blade) when a different number of isolated blades are present in the simulation. (b) Instantaneous torque per set of arms (generated by a single isolated set of arms) when a different number of isolated set of arms are present in the simulation. (c) Instantaneous torque per element (generated by a single full element) when a different number of full elements are present in the simulation.

per revolution. From Figures 11(a) and 11(b) it is also seen that the maximum values generated by three isolated blades (IB-3) and three isolated set of arms (IA-3) is actually greater than the peak values generated by one or two blades (IB1, IB-2) or sets of arms (IA-1, IA-2). As stated above, this fact is due (beside the merely contribution of more than one part) to the greater wake generated by the presence of multiple parts, which effectively increases the performances in the downwind zone. As stated in Table 2, the average performance coefficient for both isolated blades and set of arms grows with the number of elements involved.

However, when it comes to the full elements, passing to FE-2 and FE-3, the curves of Figure 11(c) show that maximum instantaneous torque is actually smaller than the one generated by only one element FE-1. This is due to a stronger wake that compromises the performance of each single element in 
TABle 1: (a) Average $C_{P}$ generated during one revolution by one isolated blade (IB-1) and one isolated set of arms (IA-1), along with theoretical (IB-1 plus IA-1) and actual full element (FE-1) results. (b) Average vorticity magnitude in a vertical plane behind the turbine, orthogonal to the free stream velocity (see Figure 10(c)).

(a)

\begin{tabular}{lccc}
\hline Case & $\begin{array}{c}\text { Average } C_{P} \\
\text { Upwind } \\
\text { zone }\end{array}$ & $\begin{array}{c}\text { Downwind } \\
\text { zone }\end{array}$ & $\begin{array}{c}\text { Full } \\
\text { revolution }\end{array}$ \\
\hline IB-1 & 0.220 & -0.024 & $\mathbf{0 . 0 9 8}$ \\
IA-1 & 0.096 & -0.021 & $\mathbf{0 . 0 3 8}$ \\
THEOR & $\mathbf{0 . 3 1 6}$ & $-\mathbf{0 . 0 4 5}$ & $\mathbf{0 . 1 3 6}$ \\
FE-1 & 0.261 & -0.027 & $\mathbf{0 . 1 1 7}(-14 \%)$ \\
\hline
\end{tabular}

(b)

\begin{tabular}{lcc}
\hline IB-1 & IA-1 & FE-1 \\
\hline $0.5621 / \mathrm{s}$ & $0.4451 / \mathrm{s}$ & $0.9131 / \mathrm{s}$ \\
\hline
\end{tabular}

TABLE 2: Average performance coefficient on one revolution for all the considered cases.

\begin{tabular}{lcc}
\hline Case & Average $C_{P}$ & $\begin{array}{c}\text { \% difference } \\
\text { compared to the } \\
{ }^{*}-1 \text { case }\end{array}$ \\
\hline IB-1 & 0.098 & $+118 \%$ \\
IB-2 & 0.214 & $+276 \%$ \\
IB-3 & 0.369 & \\
IA-1 & $\mathbf{0 . 0 3 8}$ & $+\mathbf{1 1 3 \%}$ \\
IA-2 & $\mathbf{0 . 0 8 1}$ & $+\mathbf{2 8 9 \%}$ \\
IA-3 & $\mathbf{0 . 1 4 8}$ & $+120 \%$ \\
FE-1 & 0.117 & $+121 \%$ \\
FE-2 & 0.258 & \\
FE-3 & 0.259 & \\
\hline
\end{tabular}

both the upwind and downwind zones. With respect to the case FE-2, the wakes of each rotating element in case FE3 are separated (azimuthally) by $120^{\circ}$ instead of $180^{\circ}$. In the downwind zone each element encounters a wake three times per revolution instead of two times (FE-2) or one time (FE-1).

As stated in Table 2, the average performance coefficient reaches a maximum with two elements (FE-2), and then remains substantially unchanged even if a third element is added (FE-3).

To further enhance the understanding of the previous results, the behavior of a single part (or element) when other parts (or elements) are present is shown in Figure 12 and Table 3. Their efficiencies will be compared to the ones obtained when they operate alone. In the next plots, the instantaneous torque per single part (or per element) will be shown when one, two or three parts (or elements) are present in the simulation.

Figure 12(a) shows the instantaneous torque per blade in cases IB-1, IB-2, IB-3. Similarly, Figure 12(b) shows the torque per set of inclined arms in cases IA-1, IA-2 and IA-3. Finally, Figure 12(c) shows the torque per full element in cases
TABLE 3: Average performance coefficient per element on one revolution, when different numbers of elements are present in the simulation.

\begin{tabular}{|c|c|c|c|}
\hline \multirow[b]{2}{*}{ Case } & \multicolumn{3}{|c|}{ Average $C_{P}$ per element } \\
\hline & $\begin{array}{c}\text { Upwind } \\
\text { zone }\end{array}$ & $\begin{array}{c}\text { Downwind } \\
\text { zone }\end{array}$ & $\begin{array}{c}\text { Full } \\
\text { revolution }\end{array}$ \\
\hline FE-1 & 0.261 & -0.027 & 0.117 \\
\hline FE-2 & 0.215 & 0.042 & $\mathbf{0 . 1 2 9}(+10 \%)$ \\
\hline FE-3 & 0.152 & 0.020 & $\mathbf{0 . 0 8 6}(-26 \%)$ \\
\hline
\end{tabular}

FE-1, FE-2, FE-3; the latter is the actual three-element rotor configuration.

The average values on one revolution of the performance coefficients are shown in Table 3. Partial average values, in the upwind and downwind zones, are also reported.

It is clearly seen that a rotor with two elements (FE-2) has an average performance coefficient per element higher than the single element case (FE-1). Passing from case FE-1 to case FE-2, larger wake effects tend to decrease the per element efficiency in the upwind zone; at the same time the efficiency is increased significantly in the downwind zone. The result is the greatest average value per element achieved by $C_{P}(+10 \%$ in case FE-2 compared to the case FE-1).

Nonetheless, when the number of elements is increased from two (FE-2) to three (FE-3) the even larger wake effects generated by the presence of all the turbine elements tend to decrease the overall per element efficiency in both the upwind and downwind zones. The result is the worst average value of $C_{P}(-26 \%$ in case $\mathrm{FE}-3$ in comparison to the case FE- 1$)$.

Wakes of all the different configurations of Figure 9 are depicted in the set of Figure 13(a) in terms of scalar fields of the $X$-velocity component (same direction as $U_{\infty}$ ) taken at the mid-hub height. Example of velocity vector fields in planes' orthogonal to the turbine axis is reported in Figure 13(b). Examples of streamlines and vorticity magnitude maps are shown in Figure 13(c).

\section{Results with Different Turbine Radii and Arm Chords}

A final investigation is presented here that emphasizes the role of two geometrical parameters: the turbine radius and the arm chord length.

Figure 14(a) shows that an optimal radius exists that maximizes the peak of $C_{P}$ with TSR when all the geometrical and aerodynamic parameters are fixed, for a given wind velocity. Figure 14(b) shows that lesser chord lengths of the inclined arms bring performance improvements (at higher TSRs). This is clearly due to the reduction of parasite drag.

One of the aims of the paper is to bring up the above concepts, in particular by decreasing the inclined arm chord; starting from a baseline case, one obtains an efficiency improvement. The assessment of efficiency trends with varying inclined arm chords and blade pitching angles is the scope of a future article. 


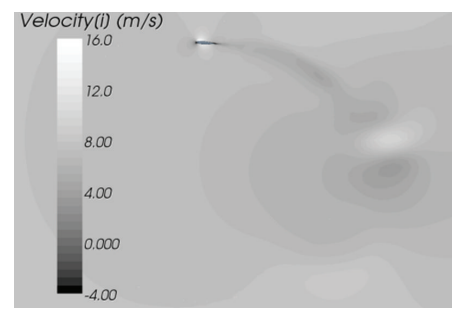

IB-1

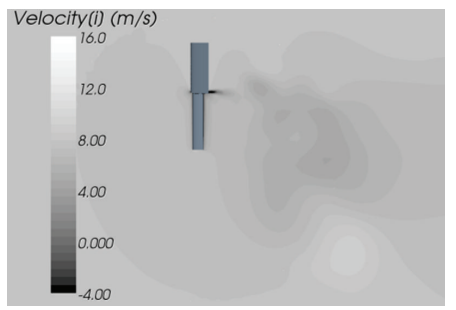

IA-1

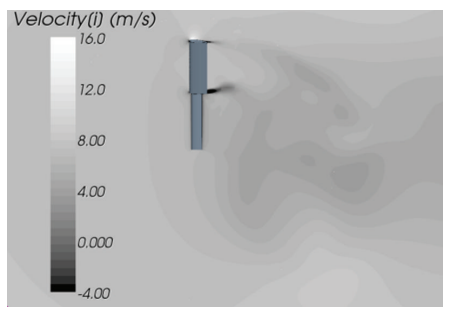

FE-1

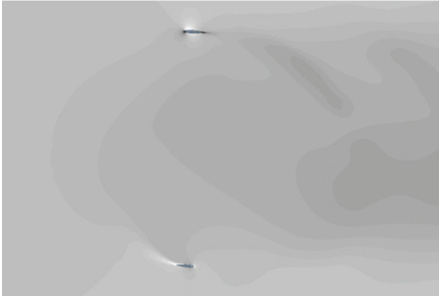

IB-2

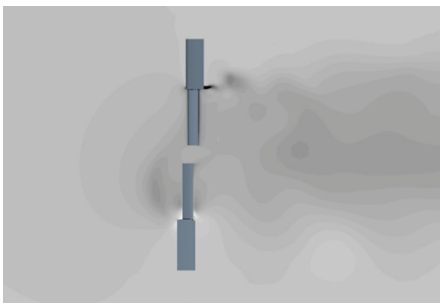

IA-2

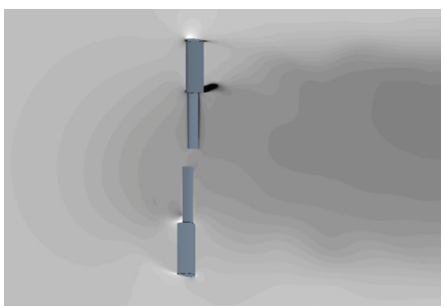

FE-2

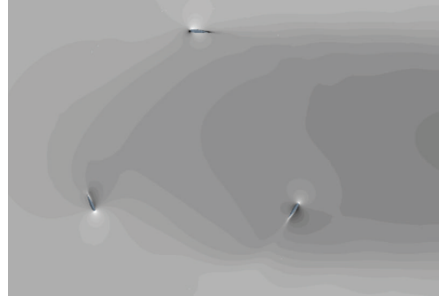

IB-3

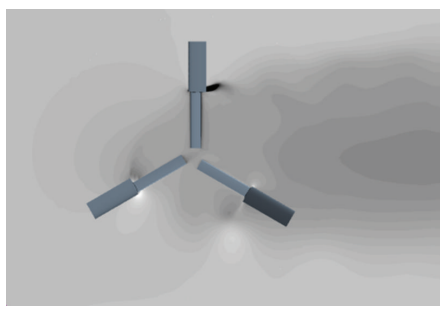

IA-3

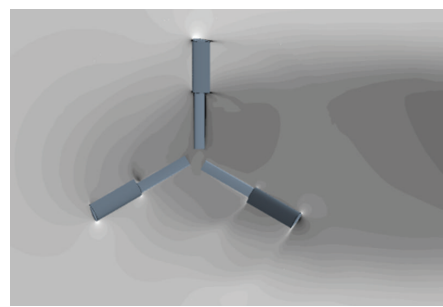

FE-3

(a)
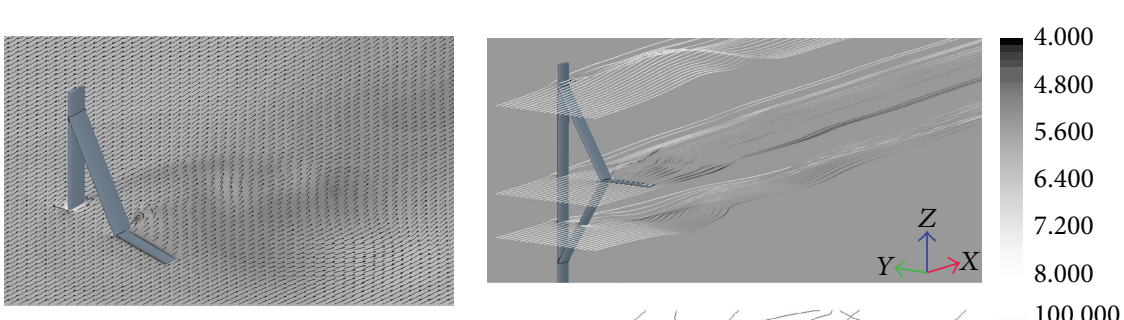

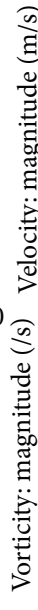
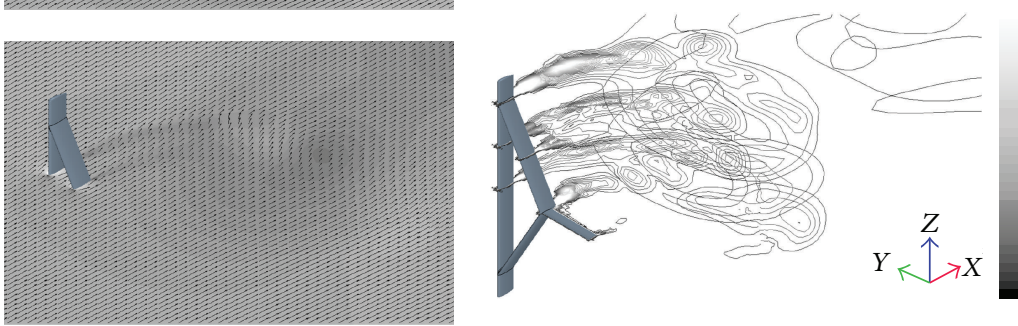

100.000

80.000

60.000

40.000

20.000

0.000

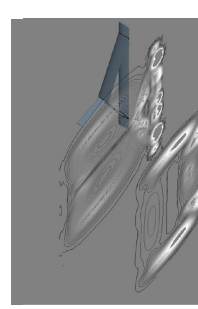

25.000

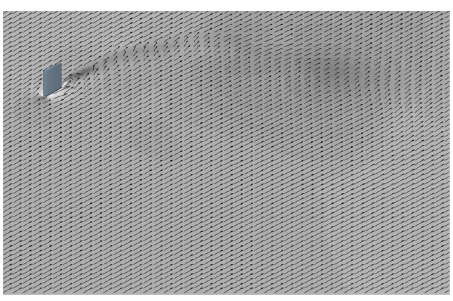

(b) (c)

FIGURE 13: (a) Scalar fields of $X$-velocity component, which has the same direction of the asymptotic wind velocity (wind blowing from left to right), taken at hub height. First row shows simulation results for one or multiple blades. Second row shows results for one or multiple arms. Third row shows results for one or multiple turbine elements. The blockage effect of the turbine is clearly seen, especially in the downwind zone, being enhanced as the number of elements grown. The local presence of reverse flow is also visible. (b) Velocity vector fields in a plane orthogonal to the turbine axis. (c) Streamlines (top) and vorticity magnitude maps in planes orthogonal to the turbine axis (middle) and in planes orthogonal to the free stream velocity, in the downwind zone (bottom). 


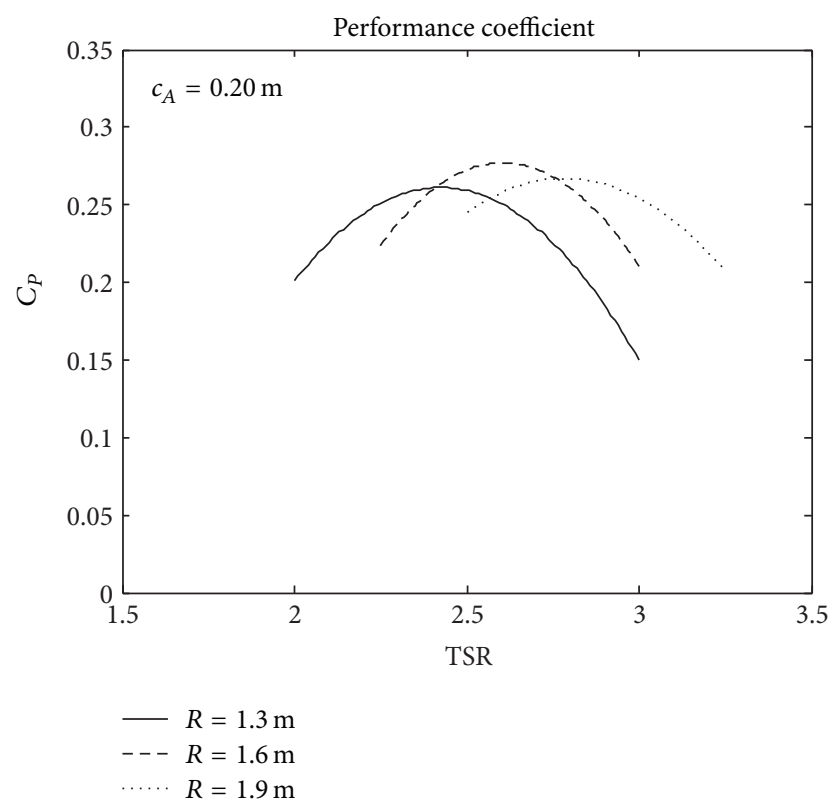

(a)

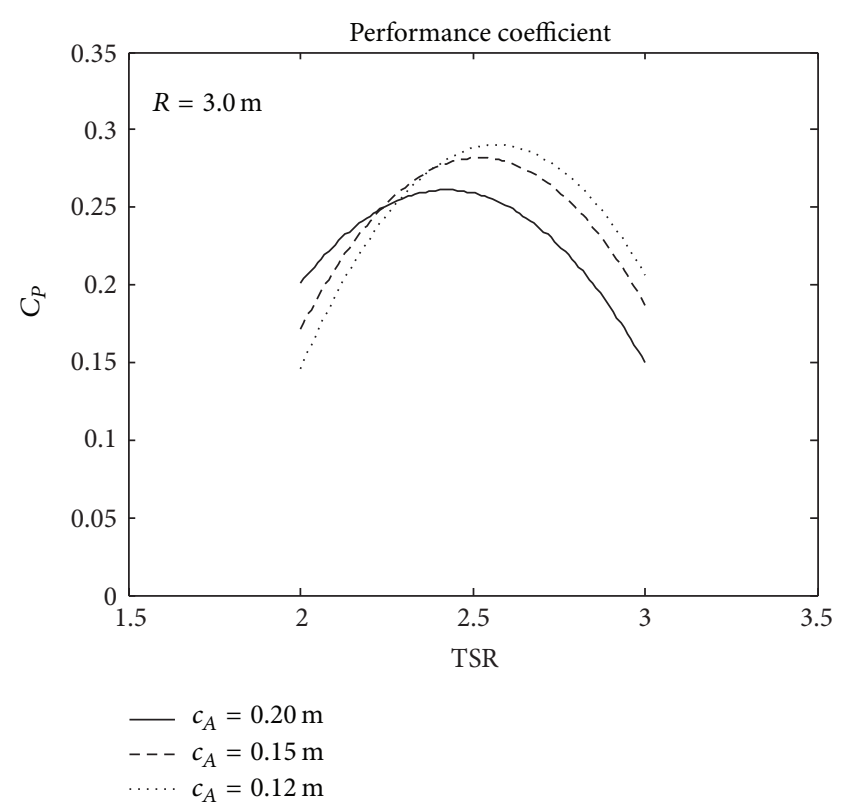

(b)

Figure 14: (a) Performance coefficient against tip speed ratio for different turbine radii (FE-3). (b) Performance coefficient against tip speed ratio for different arm chord lengths (FE-3).

\section{Conclusions}

Performances and flow fields for a wind turbine with unconventional arms geometry were numerically computed solving the three-dimensional RANS equations with SST K-Omega turbulence model. Initially, results for a single blade-plusarms element were presented, and it has been shown that the presence of inclined/profiled arms actually increases the average value (per revolution) of performance coefficient. Also, the larger wake effects generated by the presence of the arms seem to increase the performance of the positivepitched blade in the downwind zone by reducing its local incidence angle. These positive results are further enhanced as the number of turbine elements is brought to two. When the number of turbine elements is then set to the original value of three, the very large blockage effect on the incoming wind flow is clearly felt on the turbine performances.

The number of blades is just one of the many parameters that can be investigated. Results can change greatly if parameters such as turbine radius, arm chord, and blade pitch are changed. Arm chord, especially, is a fundamental variable as a small reduction of this parameter could lead to minor blockage effect on the flow while still guaranteeing a positive torque during the revolution.

\section{Conflict of Interests}

The authors declare that there is no conflict of interests regarding the publication of this paper.

\section{References}

[1] I. Paraschivoiu, Wind Turbine Design: With Emphasis on Darrieus Concept, Polytechnic International Press, Montréal, Canada, 2002.

[2] C. A. Consul, R. H. J. Willden, E. Ferrer, and M. D. McCulloch, "Influence of solidity on the performance of a cross-flow turbine," in Proceedings of the 8th European Wave and Tidal Energy Conference (EWTEC '09), Uppsala, Sweden, 2009.

[3] K. Hamada, T. Smith, N. Durrani, N. Qin, and R. Howell, "Unsteady flow simulation and dynamic stall around vertical axis wind turbine blades," in Proceedings of the 46th AIAA Aerospace Sciences Meeting and Exhibit, AIAA 2008-1319, Reno, Nevada, January 2008.

[4] R. Howell, N. Qin, J. Edwards, and N. Durrani, "Wind tunnel and numerical study of a small vertical axis wind turbine," Renewable Energy, vol. 35, no. 2, pp. 412-422, 2010.

[5] C. J. Simão Ferreira, A. van Zuijlen, H. Bijl, G. van Bussel, and G. van Kuik, "Simulating dynamic stall in a two-dimensional vertical-axis wind turbine: verification and validation with particle image velocimetry data," Wind Energy, vol. 13, no. 1, pp. $1-17,2010$.

[6] P. R. Spalart and S. R. Allmaras, "A one-equation turbulence model for aerodynamic flows," in Proceedings of the 30th Aerospace Sciences Meeting and Exhibit, AIAA, Reno, Nev, USA, 1992.

[7] F. R. Menter, "Zonal two equation k- $\omega$ turbulence models for aerodynamic flows," AIAA Paper 93-2906, 1993.

[8] M. R. Castelli, G. Simioni, and E. Benini, Numerical Analysis of the Influence of Airfoil Asymmetry on VAWT Performance, Engineering and Technology 61, World Academy of Science, 2012. 
[9] M. R. Castelli and E. Benini, "Effect of blade inclination angle on a darrieus wind turbine," Journal of Turbomachinery, vol. 134, no. 3, Article ID 031016, 10 pages, 2011.

[10] M. Raciti Castelli, S. de Betta, and E. Benini, "Effect of blade number on a straight-bladed vertical-axis Darreius wind turbine," World Academy of Science, Engineering and Technology, vol. 64, pp. 206-212, 2012.

[11] M. Raciti Castelli, A. Englaro, and E. Benini, "The Darrieus wind turbine: proposal for a new performance prediction model based on CFD," Energy, vol. 36, no. 8, pp. 4919-4934, 2011.

[12] E. Amet, T. Maître, C. Pellone, and J.-L. Achard, "2D numerical simulations of blade-vortex interaction in a darrieus turbine," Journal of Fluids Engineering, vol. 131, no. 11, Article ID 111103, 15 pages, 2009.

[13] I. S. Hwang, Y. H. Lee, and S. J. Kim, "Optimization of cycloidal water turbine and the performance improvement by individual blade control," Applied Energy, vol. 86, no. 9, pp. 1532-1540, 2009.

[14] D. P. Coiro, G. Troise, F. Scherillo, A. de Marco, and U. Maisto, "Experimental tests of GEM-Ocean's kite, an innovative patented submerged system for marine current energy production," in Proceedings of the 3rd International Conference on Clean Electrical Power: Renewable Energy Resources Impact (ICCEP '11), pp. 223-230, Capri, Italy, June 2011.

[15] D. P. Coiro, F. Nicolosi, A. de Marco, S. Melone, and F. Montella, "Dynamic behavior of novel vertical axis tidal current turbine: numerical and experimental investigations," in Proceedings of the 15th International Offshore and Polar Engineering Conference (ISOPE '05), Seoul, Republic of Korea, June 2005.

[16] D. P. Coiro, F. Nicolosi, A. de Marco, S. Melone, and F. Montella, "Flow curvature effects on dynamic behaviour of a novel vertical axis tidal current turbine: numerical and experimental analysis," in Proceedings of the ASME 24th International Conference on Offshore Mechanics and Arctic Engineering (OMAE'05), pp. 601609, Halkidiki, Greece, June 2005.

[17] R. Courant, K. Friedrichs, and H. Lewy, "Über die partiellen differenzengleichungen der mathematischen physik," Mathematische Annalen, vol. 100, no. 1, pp. 32-74, 1928.

[18] International Electrotechnical Commission, IEC International Standard 61400-1, 3rd edition, 2007.

[19] L. Merola, “The S.Co.P.E. Project,” 2006. 

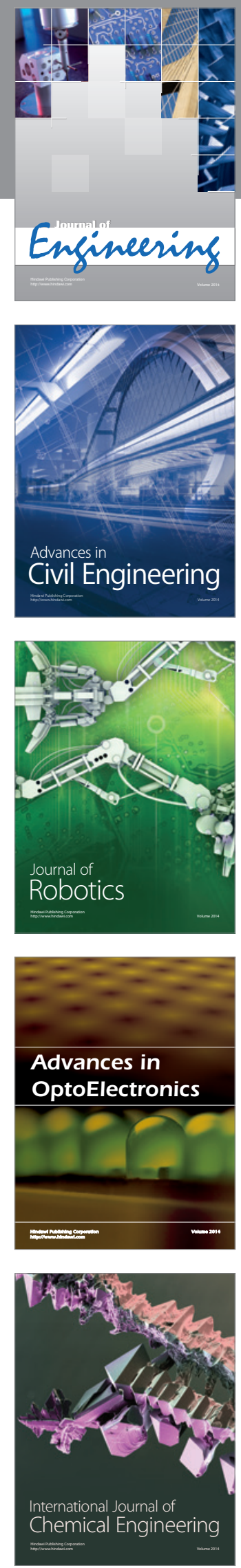

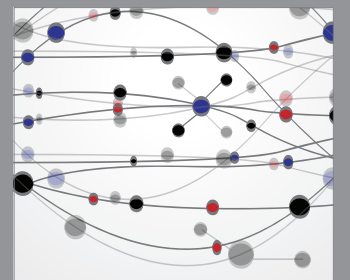

The Scientific World Journal
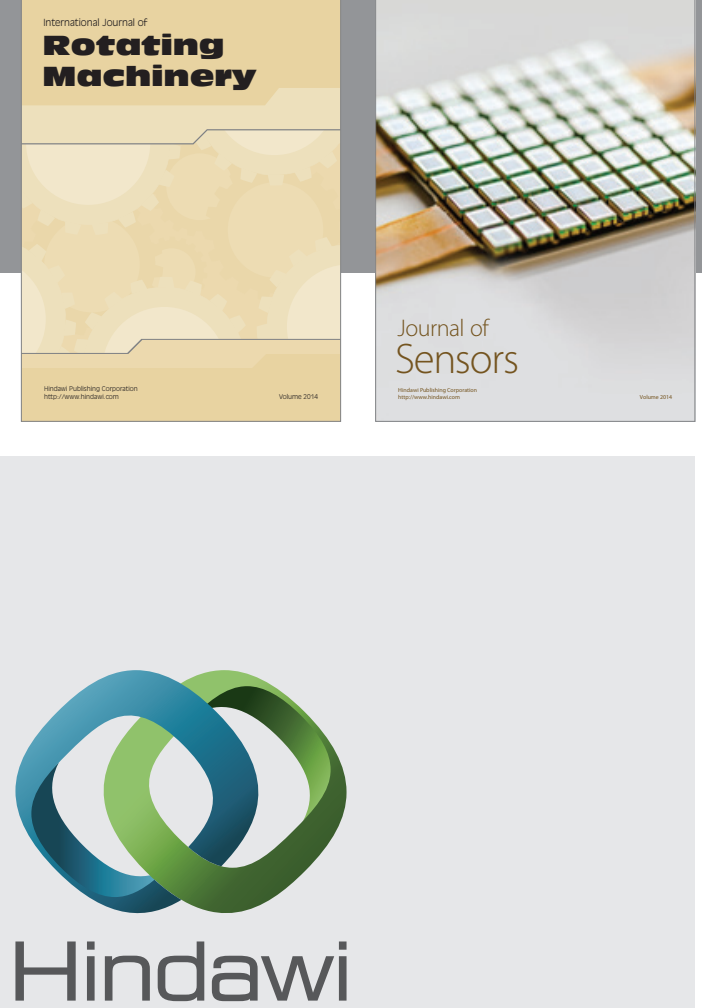

Submit your manuscripts at http://www.hindawi.com
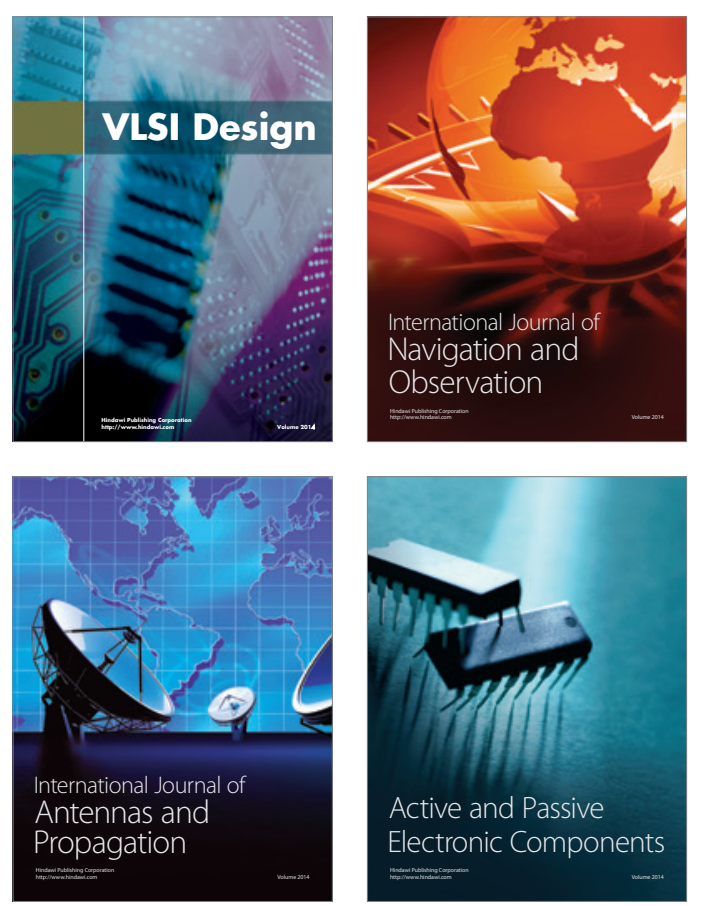
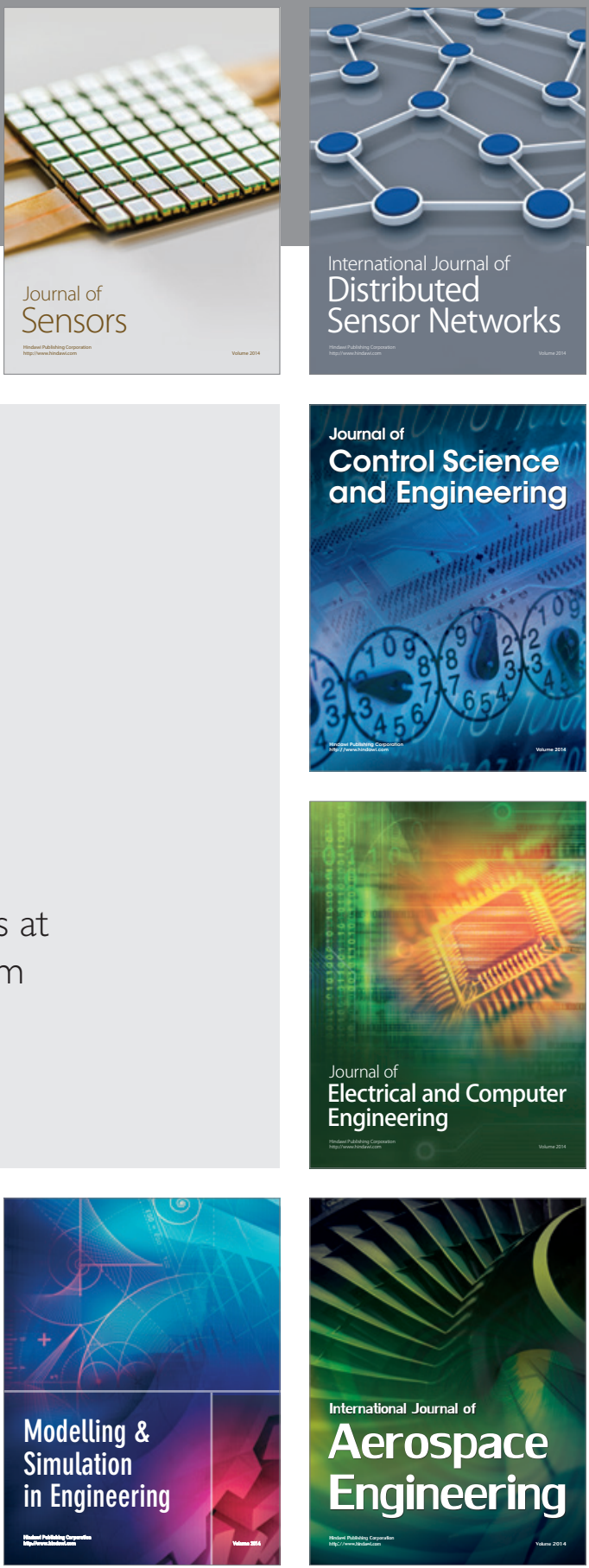

Journal of

Control Science

and Engineering
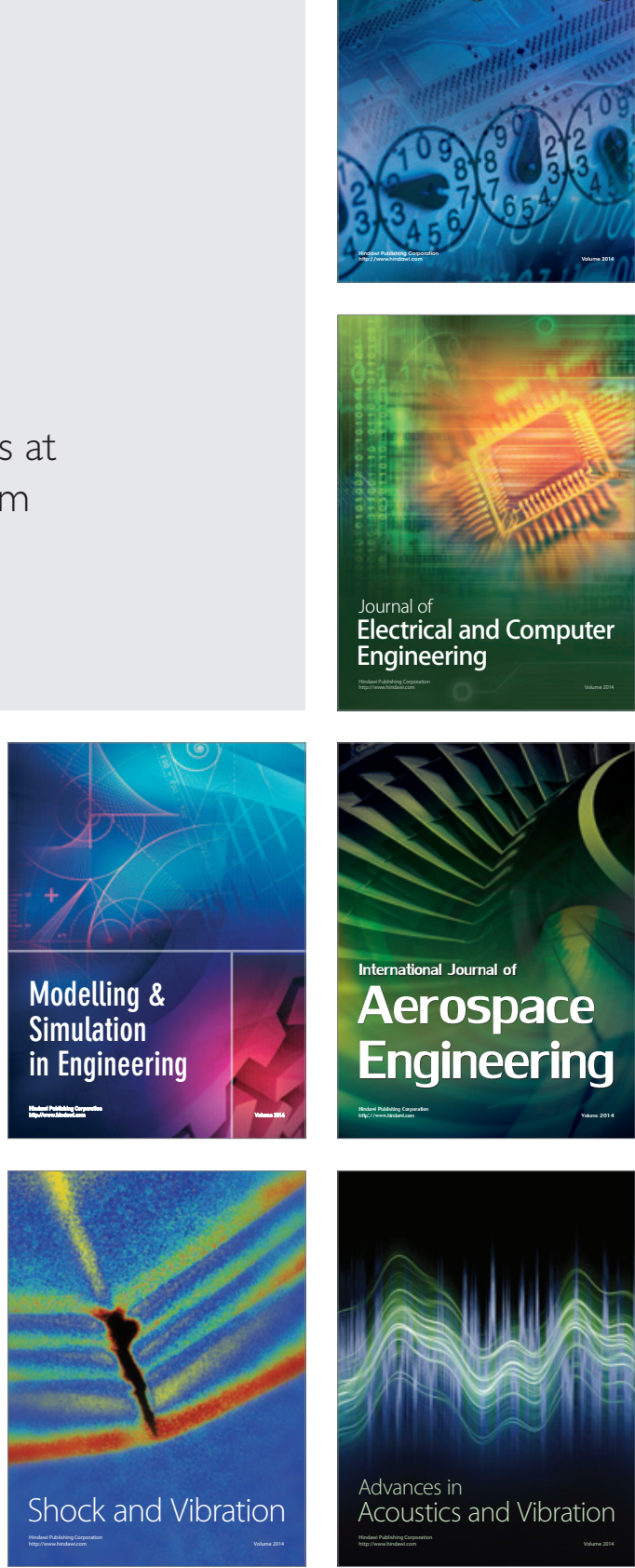